\title{
GPPS-BJ-2019-0143
}

\section{Modeling of jet fuel spray impingement in a wind tunnel}

\author{
Songyang Li \\ AECC Commercial Aircraft Engine Co., Itd. \\ lisongyang@acae.com.cn \\ Shanghai, China
}

\author{
Zhengjun Wang \\ AECC Commercial Aircraft Engine Co., Itd. \\ zhengjun_wx@163.com \\ Shanghai, China
}

\author{
Pengfei Zou \\ AECC Commercial Aircraft \\ Engine Co., Itd. \\ perfyjoe@163.com \\ Shanghai, China
}

\author{
Yuyang Li \\ Shanghai Jiaotong University \\ yuygli@sjtu.edu.cn \\ Shanghai, China
}

\author{
Tianyu Li \\ Shanghai Jiaotong University \\ Tianyu93@sjtu.edu.cn \\ Shanghai, China
}

\begin{abstract}
With the growing awareness of pollutant emissions from aero-engine combustor, fuel atomization system has been studied extensively. Fuel spray spatial distribution, droplet size, as well as fuel/air mixing play important roles in improving combustion performance. Air blast atomizer is one kind of the systems used in aero-engine combustors which involves shear driven pre-filming atomization. It creates a thin film of fuel along a solid surface, and then subjecting that film to shear from high-velocity air flow to achieve a secondary atomization. In this process, the spray wall interaction and hydrodynamic of the film formed on the filmer wall accelerate the atomization and the mixture of fuel and air, and also directly impact the later pre-filming atomization. For this reason, various researchers have studied the spray-wall interaction and the droplet formation after the impingement in the presence of a cross flow.

In this paper, we use two spray-wall interaction models to simulate experiments performed by us. In the experiment, liquid jet was injected from a plain nozzle placed at the top of a wind tunnel, and droplets were shed from the jet surface due to primary atomization in the presence of high shearing cross flowing air. Fuel droplets then hit the wall to form a film, while secondary droplets were splashed. This process is simulated under different air flow velocities and jet fuel flow rates to evaluate the models' prediction accuracy. The assessment is done by comparing the droplet sizes and film thickness downstream of the tunnel. The calculated results show in general reasonable agreement with the measurement data.
\end{abstract}

\section{INTRODUCTION}

Spray impingement on walls occurs in a wide range of situations, including gas turbines, internal combustion engine, spray cooling systems and many other applications in industry. In aircraft engine, air blast atomizer is widely implemented in several combustion systems, such as CFM56, CF6, GE90 and latest types, too (Mongia, 2001). It is one kind of pre-filming atomization system, which can stabilize highintensity combustion process for clean and efficient combustion. A typical air blast atomizer, so-called swirl cup, is composed of venturi flare, swirler and fuel nozzle. Liquid spray is first generated by a nozzle located in the center of the swirl cup, which impinges on the wall of the venturi to generate more droplets and also form an annular fuel film. This film separates from the venturi tip, and is reatomized by the counter-rotating flow. The behavior of the impinging spray has a great influence on the dispersion of the fuel, the evaporation and mixture formation process, and furthermore on the combustion processes, including the formation of the exhaust emissions of unburnt hydrocarbons (HC) and soot particles (Hsiao, 2003). Therefore, it is important to understand the spray-wall interaction behavior and the fuel dispersion process on the wall.

During this spray impingement process, several types of outcome have been observed: stick, spread, rebound, breakup and splash. Several models have been proposed for CFD simulation by previous researchers (Chaussonnet 2014). Two basic issues need to be addressed for modeling these processes. The first one is to establish regime transition criteria for predicting which regime occurs under given conditions. The second one is to quantitatively estimate the droplet characteristics after the impingement, including the 
droplet velocity magnitude and its direction for the rebound regime, the fraction of the mass deposited on the wall and the size and velocity distributions of the secondary droplets for the splash/breakup regime (Moreira, 2010).

Naber and Reitz developed a single droplet impact model serving a multi-dimensional model (Naber et al., 1988). Three forms of impact regimes were involved such as stick, reflect and wall jet, which assumed that the droplets move tangentially along the surface after impact. Mundo considered two regimes in his model, including deposition and splash (Mundo et al., 1997; Mundo et al., 1998). A disintegration parameter $\mathrm{K}$ was used to determine the regime transition criteria, which was originally introduced by Stow and Hadfield to quantity the impact energy (Stow et al., 1981). One of most widely used model was proposed by Bai and Gosman. Seven regimes of drop impingements with detailed regime transition criteria and droplets' post-impact characteristic were considered. The wall status was treated as dry wall and wet wall, respectively. Adhesion and splash were considered in dry wall status while rebound, spread and splash were included in the wet wall status (Bai and Gosman, 1995; Bai et al., 2002). Another popular model was developed by O'Rourke and Amsden. They implemented the model into KIVA, which is a famous multi-dimensional computational program for internal combustion engine. Not only the spraywall interaction but also the hydrodynamic of film were considered in the model (O'Rourke and Amsden, 1996; O'Rourke and Amsden, 2000).

In previous study, these models have emerged for the simulation of both diesel and gasoline impinging sprays usually happened in port-injected engines. The present paper focuses on jet fuel spray impingement occurred in the prefilming atomization of air blast atomizer. Two impingement models, including Bai-Gosman model and O'Rourke-Amsden model, are chosen for this application. The models are assessed by simulating experiments on a jet in cross flow spray impingement in a wind tunnel made in our project. Several cases with different air flow velocity and fuel flow rates are considered. The assessment is done by comparing the droplet sizes and film thickness downstream of the tunnel. A brief description of the two models, the testing and modeling setup, as well as the comparison of measured and predicted data are provided in the following section.

\section{SPRAY IMPINGEMENT MODEL}

In this section, the basic elements of the two spray-wall interaction models are described, respectively.

\section{Regime Transition Criteria}

The impact energy and the wall temperature are first order parameters which are used to characterize different regimes. Second order parameters are the presence of a liquid film, the surface roughness and the system wettability.

The Bai-Gosman model considers four impingement regimes: stick, rebound, spread and splash, as shown schematically in Figure 1. The vertical axis represents the Weber number of incident particles, We, defined by $\rho V_{r}^{2} d_{I} / \sigma$, and the horizontal axis stands for the thickness of wall-film. The existence of these regimes depends on properties of the impinging droplets and the impingement surface, including whether the wall is dry or wetted (Bai and Gosman, 1995). In their model, only the wall conditions at sub-boiling temperatures are considered. In the case of a dry wall, rebound has not been observed in previous experiments, so the rebound regime is not involved. For simplification, it is assumed that sharp boundaries are existed between different regimes and both stick and spread regimes are treated as one regime called 'adhesion'.

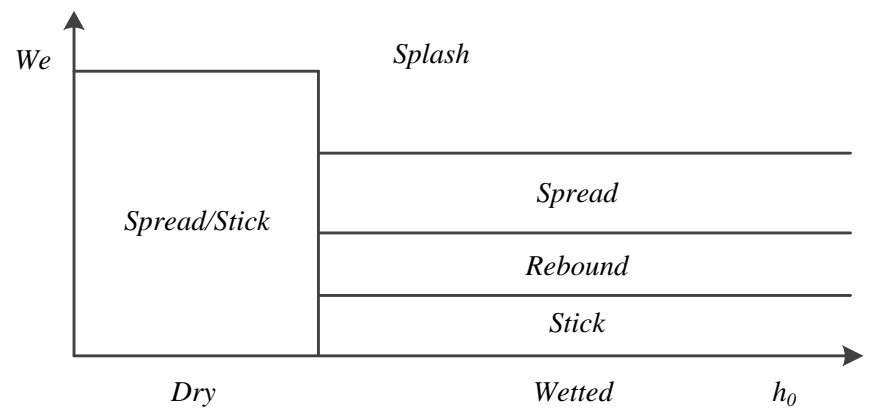

\section{Figure 1 Simplified Decision Chart for Wall Interaction Criterion in Bai-Gosman model}

Quantitative criteria for the regime transition in both dryand wet-wall status and at sub-boiling temperatures are presented in Table 1 below (Bai et al., 2002). Webber number is chosen as the threshold parameter. It can be observed that during the transition from spread to splash, the critical $W e$ is a function of droplet Laplace number, $L a$, which is defined by $\rho \sigma d_{I} / \mu^{2}$. Laplace number measures the relative importance of surface tension and viscous forces acting on the liquid. Note that the coefficients are different between dry and wet cases due to the surface roughness effect. It is assumed that a wetted surface behaves as a very rough dry wall.

\section{Table 1 Regime transition conditions of Bai- Gosman model}

\begin{tabular}{|l|l|l|}
\hline Wall status & Regime transition state & Critical Weber number \\
\hline \multirow{2}{*}{ Dry } & $\begin{array}{l}\text { Adhesion(Stick/Spread) } \\
\rightarrow \text { Splash }\end{array}$ & $W e_{\text {crit }}=2630 \mathrm{La}^{-0.183}$ \\
\hline \multirow{3}{*}{ Wet } & Stick $\rightarrow$ Rebound & $W e_{\text {crit }}=2$ \\
\cline { 2 - 3 } & Rebound $\rightarrow$ Spread & $W e_{\text {crit }}=20$ \\
\cline { 2 - 3 } & Spread $\rightarrow$ Splash & $W e_{\text {crit }}=1320 \mathrm{La}^{-0.183}$ \\
\hline
\end{tabular}

For O'Rourke-Amsden model, there are four regimes: stick, rebound, spread and splash, are considered as shown schematically in Figure 2 (O'Rourke and Amsden, 2000). The vertical axis represents the impact energy, $E$, and the horizontal axis stands for the wall temperature, $T_{w}$, where $T_{b}$ is the liquid boiling temperature. Below the boiling temperature of the liquid, the particles after impact can stick, spread or splash, while above the boiling temperature, they can either rebound or splash.

The transition criteria by which the regimes are partitioned are based on the impact energy and the boiling temperature of the liquid. The impact energy is defined by 


$$
E^{2}=\frac{\rho V_{r}^{2} d_{I}}{\sigma}\left(\frac{1}{\min \left(h_{0} / d_{I}, 1\right)+\delta_{b l} / d_{I}}\right)
$$

where $\rho$ is the liquid density, $V_{r}$ is the relative velocity of the impinging droplet from the wall, $d_{I}$ is the diameter of the droplet, $\sigma$ is the surface tension of the liquid, and $h_{0}$ is the film height. Besides, $\delta_{b l}$ is a boundary layer thickness, defined by $d_{I} / \sqrt{R e}$. The Reynolds number, $R e$, is defined as $\rho V_{r} d_{I} / \mu$.

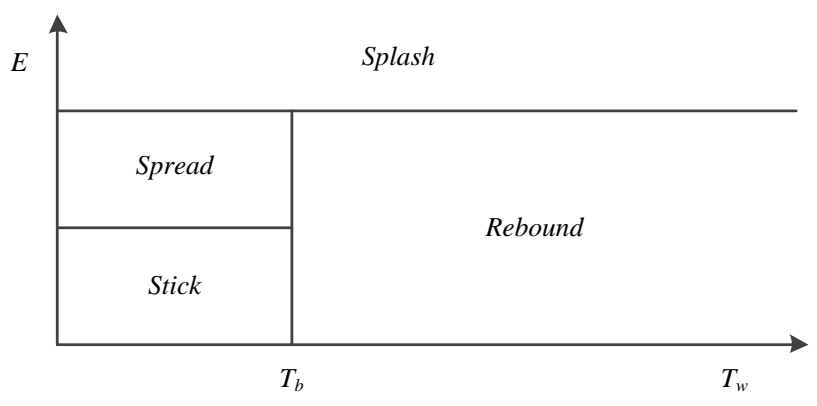

Figure 2 Simplified Decision Chart for Wall Interaction Criterion in O'Rourke-Amsden model

The regime transition criteria for both below and above boiling temperature are presented in Table 2 as below.

\section{Table 2 Regime transition conditions of O'Rourke- Amsden model}

\begin{tabular}{|l|l|l|}
\hline Wall status & Regime transition state & Critical impact energy \\
\hline \multirow{2}{*}{ Cold } & Stick $\rightarrow$ Spread & $E_{\text {crit }}=16$ \\
\cline { 2 - 3 } & Spread $\rightarrow$ Splash & $E_{\text {crit }}=57.7$ \\
\hline \multirow{2}{*}{ Hot } & Rebound $\rightarrow$ Splash & $E_{\text {crit }}=57.7$ \\
\hline
\end{tabular}

\section{Post-impingement Characteristics}

The methodologies, which are employed to determine the particles post-impingement characteristics for different regimes considered in the two models, are described below.

\section{(1) Stick}

Here the impact droplets are assumed to coalesce to form a local film, and the velocity is set equal to the wall velocity.

\section{(2) Spread}

In the spread regime, the arriving droplets also coalesce to form a local film, and the initial velocity and direction of the particles representing the film are set using a wall-jet model proposed by Naber and Reitz, where the probability of these particles' behavior having a particular direction along the surface are defined empirically (Naber et al., 1989).

\section{(3) Rebound}

For this regime, the following relation for the kinetic energy loss of a rebounding droplet is chosen in both models, initially proposed by Matsumoto (Matsumoto et al., 1970):

$$
V_{S T}=\frac{5}{7} V_{I T}
$$

$$
V_{S N}=-e V_{I N}
$$

where $V_{I T}, V_{I N}$ are the tangential and normal incident velocity components, respectively, and $V_{S T}, V_{S N}$ are the components of the rebound velocity. The normal rebound velocity is determined with the following coefficient of restitution:

$$
e=0.993-1.76 \theta_{I}+1.56 \theta_{I}^{2}-0.49 \theta_{I}^{3}
$$

where $\theta_{I}$ is the impingement angle in radians as measured according to the wall surface.

\section{(4) Splash}

For this regime, four primary quantities need to be determined, including the total secondary to impact droplet mass ratio $m_{S} / m_{l}$, as well as the secondary droplet sizes, velocities and ejection angles.

Mass Ratio - The ratio $m_{S} / m_{I}$ is believed to be influenced by a number of parameters, such as $W e, L a, \delta_{b l}$, and $h_{0}$. In BaiGosman model (Bai and Gosman, 1995), the following approximations are taken which are primarily derived from experimental observation:

$$
\frac{m_{S}}{m_{I}}=-\left\{\begin{array}{l}
0.2+0.6 \alpha, \text { for } h_{0}=0 \\
0.2+0.9 \alpha, \text { for } h_{0}>0
\end{array}\right.
$$

where $\alpha$ is a random number distributed uniformly in the interval $[0,1]$.

In O'Rourke-Amsden model, the amount of mass splashed from the wall surface is considered as a quadratic function of the splashing energy (O'Rourke and Amsden, 2000), derived from the experimental data by Mundo (Mundo et al., 1995). The splashed mass fraction is given by:

$$
\frac{m_{S}}{m_{I}}=- \begin{cases}1.8 \times 10^{-4}\left(E^{2}-E_{\text {crit }}^{2}\right), & \text { for } E_{\text {crit }}^{2}<E^{2}<7500 \\ 0.75, & \text { for } 7500<E^{2}\end{cases}
$$

Secondary Droplet Sizes - In both Bai-Gosman model and O'Rourke model, it is assumed that each incident droplet produces $p$ secondary particles, where $p \geq 1$. Each of these particles contains an equal proportion of mass, $m_{S} / p$. For each splashed particle, a different diameter is obtained by sampling a cumulative probability distribution function (PDF).

In Bai-Gosman model, it is determined that these droplet size data can be fitted by a Chi-squared distribution function (Bai et al., 2002) given by:

$$
f(d)=\frac{1}{\bar{d}} \exp \left(-\frac{d}{\bar{d}}\right)
$$

where $\bar{d}$ is the number mean diameter which is related to the volumetric mean diameter $d_{V}$ as below :

$$
\bar{d}=\frac{d_{V}}{6^{1 / 3}}=\frac{1}{6^{1 / 3}}\left(\frac{r_{m}}{N_{S}}\right)^{1 / 3} d_{I}
$$

The quantity $N_{S}$ denotes the total number of secondary droplets per splash, obtained in their previous work (Bai and Gosman, 1995) as:

$$
N_{S}=a_{0}\left(\frac{W e}{W e_{c r i t}}-1\right)
$$


where $a_{0}=5$ and $W e_{c r i t}$ is the critical Weber number for splashing.

The probability density function (7) can now be integrated to obtain a cumulative probability function, which is then used to determine the sizes of $p$ secondary droplet parcels, $d_{i}(i=1 \ldots p)$ by taking $p$ random samples.

Finally, the number of droplets $n_{i}$ in each secondary parcel $i$ is determined by requiring mass conservation:

$$
n_{i} d_{i}^{3}=\frac{r_{m} d_{I}^{3}}{p}
$$

In O'Rourke-Amsden model, it is assumed that size distributions of secondary droplets are consistent with a Nukiyama-Tanasawa size distribution (O'Rourke and Amsden, 2000):

$$
f(r)=\frac{4}{\sqrt{\pi}} \frac{r^{2}}{r_{\max }^{3}} \exp \left[-\left(\frac{r}{r_{\max }}\right)^{2}\right]
$$

where $r_{\max }$ is the droplet radius at which the PDF achieves its maximum value. The value of $r_{\max } / r_{I}$ is a function of $E$ which is approximated by:

$$
\frac{r_{\max }}{r_{I}}=\max \left(\frac{E_{c r i t}^{2}}{E^{2}}, \frac{6.4}{W e}, 0.06\right)
$$

Also, the cumulative probability function is used to calculate the sizes of secondary droplets, and the number of droplets in each secondary parcel is calculated through mass conservation.

Secondary Droplets Velocity Components - In BaiGosman model, the secondary droplet velocity fields are divided into a normal impingement component and a tangential component along the wall, so the splash velocity vector, $V_{S}$, of a secondary droplet is given by:

$$
V_{S}=V_{S T}+V_{S N}
$$

where $V_{S T}$ is assumed to dependent solely on $V_{I T}$, and $V_{S N}$ is solely depended on $V_{I N}$ as well.

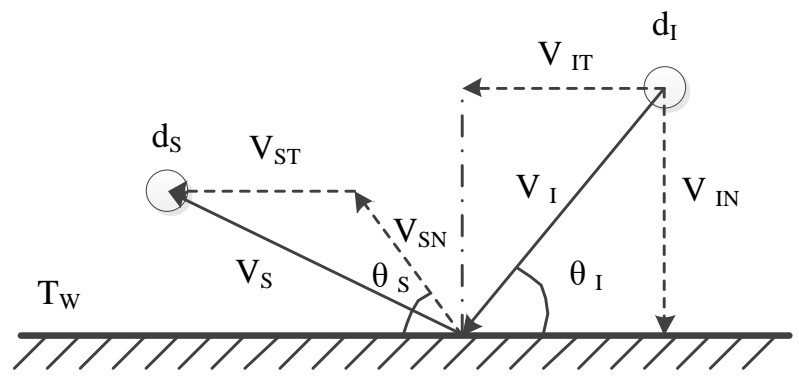

\section{Figure 3 Diagram illustrating droplet impingement onto a wall}

Note that VSN is not in general normal to the wall surface as schematically defined in Figure 3. The component VST is assumed to be related to the incident tangential velocity VIT by:

$$
V_{S T}=C_{f} V_{I T}
$$

where the friction coefficient $C_{f}$ is taken in the range [0.6, 0.8] (Bai et al., 1995) according to experimental observation.

Therefore, the secondary droplet normal velocity, $V_{S N}$, can be evaluated only by considering the nominal incident velocity $V_{I N}$, without involvement of $V_{I T}$ and $V_{S T}$. The evaluation is performed as blow: (a) The azimuthal angle, $\psi_{S, i}$, which is defined in the circumferential direction within the plane tangential to the wall, is randomly sampled in the range $[0,2 \pi]$ with an equal probability. (b) The ejection angle, $\theta_{S, i}$, is experimentally observed in the range $\left[5^{\circ}, 50^{\circ}\right]$ where an equal probability is also chosen in the model. (c) The calculation of velocity magnitude, $V_{I N, i}$, is done by considering energy conservation which yields the following equation :

$$
\frac{1}{2} \frac{m_{S}}{p}\left[\left(V_{S N, 1}\right)^{2}+\ldots+\left(V_{S N, p}\right)^{2}\right]=E_{K S}
$$

where $E_{K S}$ is the splash kinetic energy due to $V_{I N}$ only, given by:

$$
E_{K S}=E_{K I}+E_{I \sigma}-E_{D}-E_{S \sigma}
$$

in which $E_{K I}$ denotes the incident kinetic energy based on the normal incident velocity, given by:

$$
E_{K I}=\frac{1}{2} m_{I} V_{I N}^{2}
$$

$E_{I \sigma}$ is the incident droplet surface energy, $E_{S \sigma}$ is the total surface energy of splashing droplets, and $E_{D}$ is the dissipative energy loss, given by:

$$
E_{D}=\max \left(0.8 E_{K I}, \frac{W e_{c r i t}}{12} \pi \sigma d_{I}^{2}\right)
$$

Eqs. (15) and (16) are adequate to determine $V_{S N, i}$ if only one secondary particle is introduced which means $p=1$. Otherwise, when $p>1$, a size-velocity correlation derived from the experimental data (Ghadiri, 1978) is used as a supplemental equation:

$$
\left(\frac{V_{S N, 1}}{V_{S N, i}}\right) \approx \ln \left(\frac{d_{1}}{d_{I}}\right) / \ln \left(\frac{d_{i}}{d_{I}}\right) \quad(i=2 \ldots p)
$$

In O'Rourke-Amsden model, the secondary droplet normal and tangential velocities also scaled with incident drop normal and tangential velocities, respectively. The secondary droplet velocity vector, $\overrightarrow{V_{S}}$, is approximated as below according to observed distributions:

$$
\vec{V}_{S}=V_{N}^{\prime} \vec{n}+\left(0.12 V_{I N}+V_{T}^{\prime}\right)\left(\cos \psi \vec{e}_{t}+\sin \psi \vec{e}_{p}\right)+0.8 V_{I T} \vec{e}_{t}
$$

where $\vec{n}$ is the unit normal to the wall surface, $\overrightarrow{e_{t}}$ is the unit vector tangent to the wall and in the plane of $\vec{n}$ and the incident drop velocity, $\overrightarrow{e_{p}}=\vec{n} \times \overrightarrow{e_{t}}$, and $V_{N}^{\prime}, V_{T}^{\prime}$, and $\psi$ are random variables chosen by three different PDFs.

The quantity $V_{N}^{\prime}$ is the normal component of the secondary droplet velocity and is sampled from the Nukiyama-Tanasawa distribution. The quantity $V_{T}^{\prime}$ is the fluctuating component of the secondary droplet tangential velocity, chosen from the Gaussian distribution. Finally, $\psi$ denotes the angle that the fluctuating tangential velocity makes with the vector $\overrightarrow{e_{t}}$ in the plane of the wall. $\psi$ is chosen in the range $[-\pi, \pi]$ according to the distribution suggested by Naber and Reitz (Naber et al., 1989). 


\section{EXPERIMENT DESCRIPTION}

The experiments for a direct jet-fuel spray impingement inside a cross flow wind tunnel, which has a rectangular crosssection of $8 \times 25 \mathrm{~mm}^{2}$, has been conducted in Shanghai Jiaotong University. The geometry is illustrated in Figure 4. The injector with an orifice of $0.5 \mathrm{~mm}$ diameter has been positioned at the top along the centre line of the tunnel, and the injection direction is perpendicular to the air flow. The air is at atmospheric pressure and room temperature.

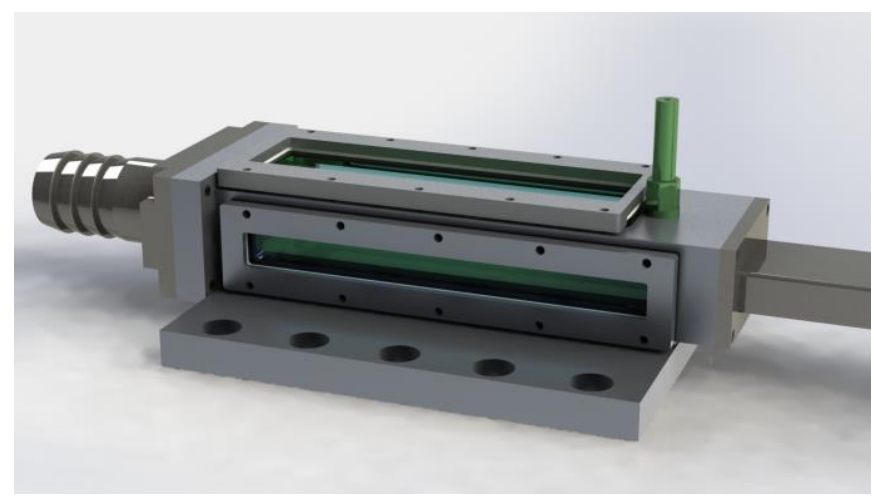

Figure 4 Experimental device for a spray impingement within cross flow wind tunnel

Six cases have been investigated in the experiments, involving mean cross flow air velocities of $75 \mathrm{~m} / \mathrm{s}$ with three different jet flow rates (Case 1, 2, 3) and mean jet flow rate of $176.71 \mathrm{ml} / \mathrm{min}$ with three different mean cross flow air velocities (Case 4, 5, 6), respectively. The inlet conditions of these cases are listed in Table 3.

\section{Table 3 Inlet conditions of experimental cases}

\begin{tabular}{|c|c|c|}
\hline \multirow[b]{2}{*}{ No. } & \multicolumn{2}{|c|}{ Inlet boundary condition } \\
\hline & $\begin{array}{c}\text { Jet flow velocity } \\
\mathrm{m} / \mathrm{s}\end{array}$ & $\begin{array}{c}\text { Air flow velocity } \\
\mathrm{m} / \mathrm{s}\end{array}$ \\
\hline 1 & 5 & 75 \\
\hline 2 & 12 & 75 \\
\hline 3 & 20 & 75 \\
\hline 4 & 15 & 50 \\
\hline 5 & 15 & 75 \\
\hline 6 & 15 & 100 \\
\hline
\end{tabular}

A high speed camera has been used to take photographs of the injected spray at regular time intervals for each case. The ensemble-averaged droplet sizes are deduced from these photographs. Measurements have been made at four different positions I, II, III, IV, which, as illustrated in Figure 5, are located in the center of the wind tunnel and lie respectively 2 , 6,10 and $14 \mathrm{~mm}$ downstream of the injector and within a horizontal bandwidth of $4 \mathrm{~mm}$.

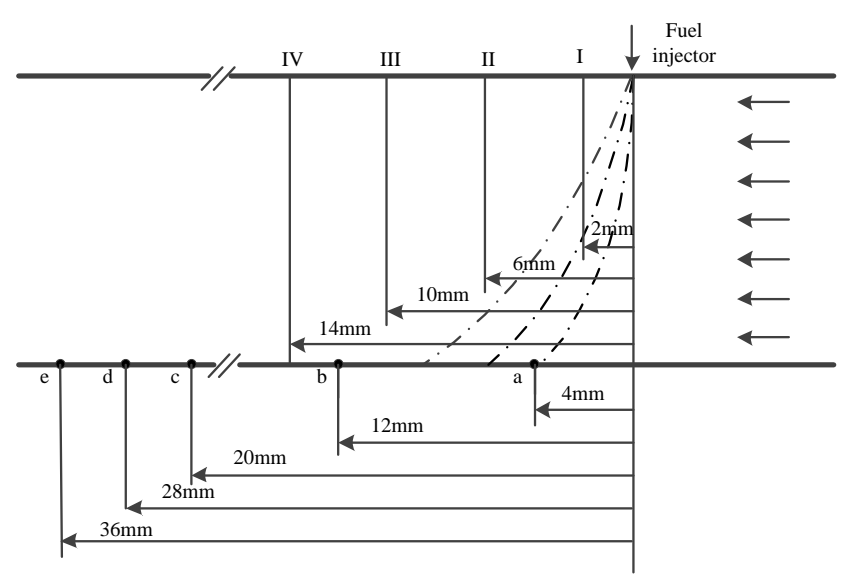

\section{Figure 5 Illustration of measurement locations for} wind tunnel experiments

The time-averaged film thickness has been measured by using the PLIF technique in the center of the wind tunnel, where the mean values have been captured at five different positions a, b, c, d, and e which lie respectively 4, 12, 20, 28 and $36 \mathrm{~mm}$ downstream of the injector on the wall surface, as illustrated in Figure 5.

In addition, the spray patterns and impact behavior have also been captured by the high speed camera.

\section{COMPUTATIONAL DETAILS}

ANSYS FLUENT 16.0 is adopted for all simulations in the presented work. Turbulent continuous phase flow field is solved by using pressure based coupled solver and droplet phase is solved through DPM approach with unsteady particle tracking method. Realizable $\mathrm{k}$ epsilon model is used along with Enhance wall treatment to model the boundary layer. The wall-film model, which is a particle-based approach for thin films first formulated by O'Rourke \& Amsden, is employed to simulate fuel film movement on the wall (O'Rourke and Amsden, 1996).

Grid independent study for spray-wall interaction and film solution has been performed with various surface mesh sizes to make sure the velocity field for both gas and droplet phase becomes grid independent. Convergences of global residuals have been achieved below $10^{-3}$ for all equations. The simulation convergence is ensured by monitoring the film mass as function of time. The results are considered converged when this monitor stabilizes.

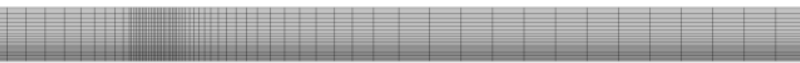

Figure 6 Mesh Distribution in mid plane

The computation domain is shown in Figure 6. Case 2, 5 and 6 are chosen for comparing the prediction of the two spray-wall interaction models against experimental results. Therefore, the inlet velocities of air and fuel are set corresponding to the test conditions, while the outlet is exposed to atmospheric condition. For side surfaces, wall boundary condition has been used. 
Primary breakup is responsible for the droplet size distribution and initial velocity at the exit of the nozzle. In this study, an in-house code for estimating plain orifice atomizer performance is adopted to caculate the initial condition of the spray nozzle. Jet fuel dissolving a fluorescent agent is taken as model fluid for spray droplet. It has density of $780 \mathrm{~kg} / \mathrm{m}^{3}$, dynamic viscosity of $0.0012 \mathrm{~kg} / \mathrm{ms}$ and surface tension of $0.024 \mathrm{~N} / \mathrm{m}$.

Both two spray-wall interaction models are compiled in FLUENT UDF language, which have been implemented into FLUENT software for modeling.

\section{RESULTS AND DISCUSSION}

The simulated results for the wind tunnel experiments of Case No. 2, 5, 6 are presented and discussed in this section. The results are obtained by using Bai-Gosman model and O'Rourke-Amsden model with above modeling set-up and boundary conditions.

Figures 7 and 8 demonstrate the snapshot and timeaveraged spray patterns for cases 5, 6, captured by high-speed camera, respectively. The time-averaged spray shapes in Figure 8 were obtained over 50 transient spray pictures. The frequency is $2 \mathrm{~Hz}$ and the pulse width of the illumination laser is $30 \mathrm{~ns}$. The spray was inclined by the high-speed cross flow, larger droplets stroke the wall surface not far downstream from the injection, while smaller droplets impinged a little further away, and the very small droplets are carried away by the cross flow before they reach the wall. It is also noticeable that the secondary droplets generated by splashing moves mainly along the wall in the downstream direction, although a few droplets are seen to move upstream.

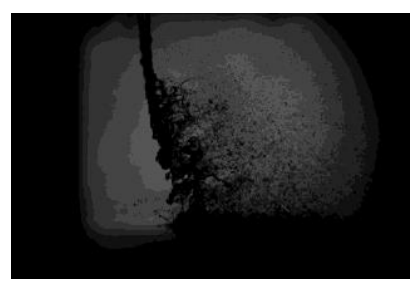

(a) Case 5

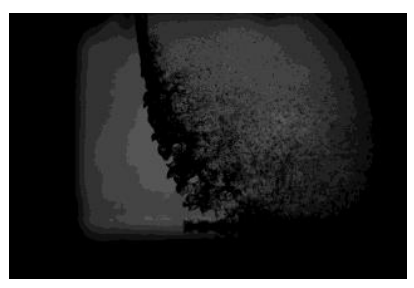

(b) Case 6
Figure 7 Snapshot of spray patterns

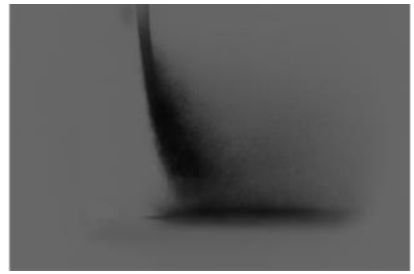

(a) Case 5

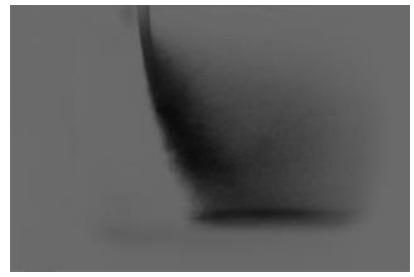

(b) Case 6
Figure 8 Time-averaged spray shapes

The above spray behaviors can also be observed in simulated results as presented in Figure 9. It can be seen that the modeling sprays, computed by both models, correspond well with the experimental pictures. It can be further observed that most smaller droplets produced by splashing move along the wall, while larger secondary droplets move to upper side.
Especially, in the snapshots of the O'Rourke-Amsden model, many large particles are observed away from the wall, whereas in these of the Bai-Gosman model many particles are sedimented to the wall. The reason is that velocity direction of the secondary particles after splashing in the Bai-Gosman model is controlled to some extent by ejection angle, $\theta_{S}$, as shown in Figure 3, which is chosen between $5^{\circ}$ and $50^{\circ}$, whereas in the O'Rourke-Amsden model, the limitation is not existed, and velocity direction is controlled by $V_{N}^{\prime}$ and $V_{T}^{\prime}$, as shown in Eq. 20. Therefore, in the O'Rourke-Amsden model, large particles have more chance to have large ejection angle.

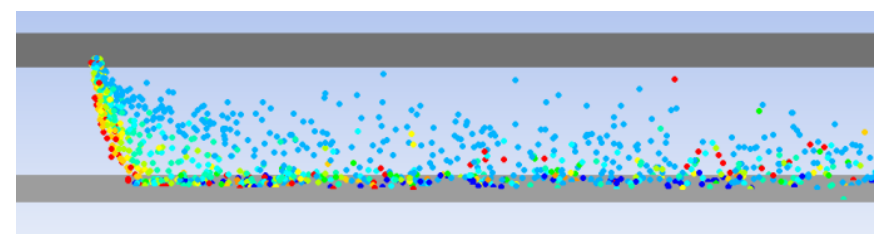

(a) Case 5 by Bai-Gosman model

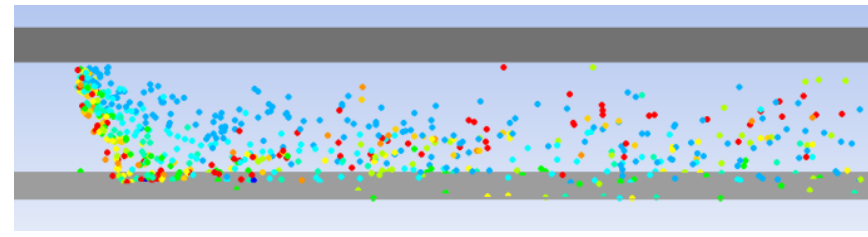

(b) Case 5 by O'Rourke-Amsden model

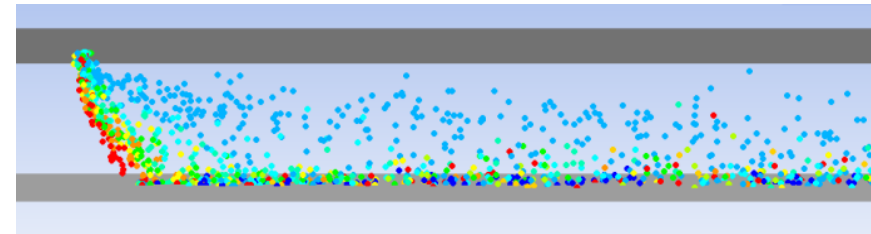

(c) Case 6 by Bai-Gosman model

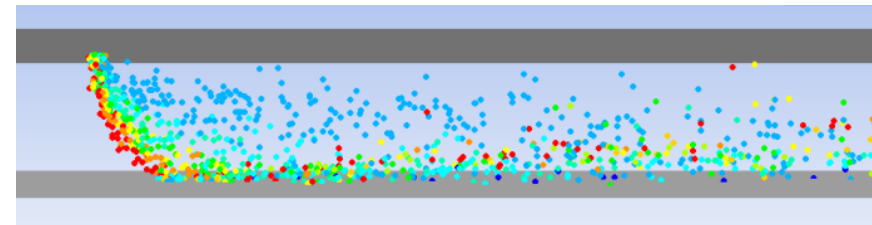

(d) Case 6 by O'Rourke-Amsden model

Particle Diameter $(\mu \mathrm{m})$

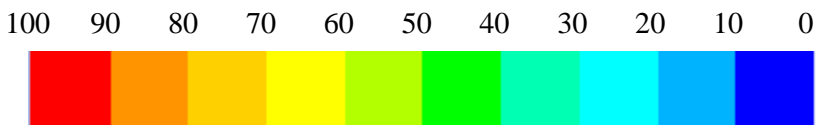

Figure 9 Predicted transient spray patterns at $0.5 \mathrm{~s}$ after initial injection

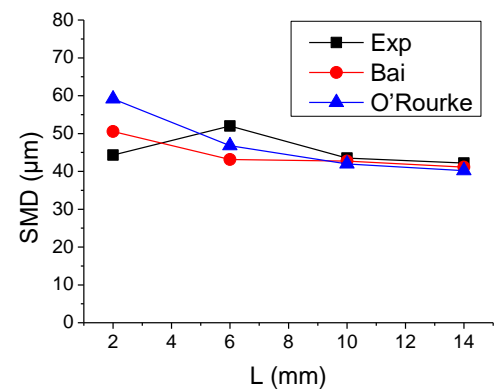

(a) Case 2 


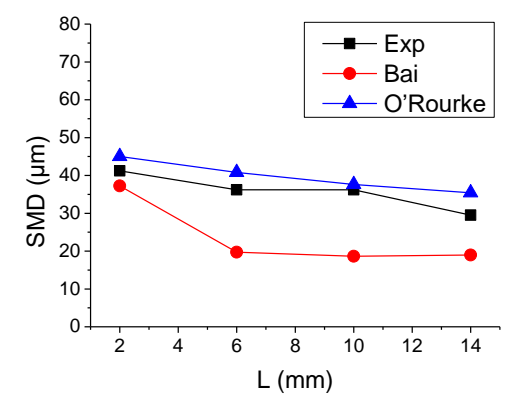

(b) Case 5

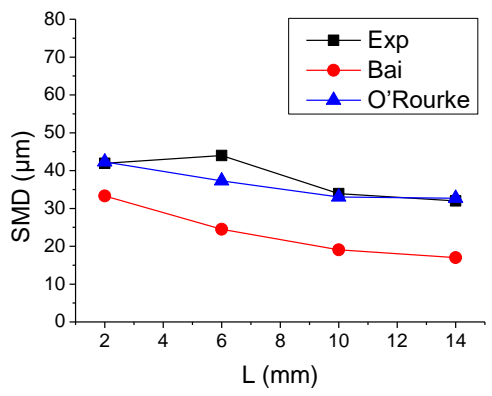

(c) Case 6

\section{Figure 10 Measured and predicted droplet mean sizes by both models}

The droplet mean sizes obtained from measurement and simulation at locations I, II, III and IV are plotted in Figure 10 for the three cases. Here the curves with black square, blue triangular and red circle symbols stand for the measured, calculated by Bai-Gosman model and O`Rourke-Amsden model respectively. Both the measured and calculated data on droplet sizes were obtained by taking into account all the droplets passing through the photographic measurement slice (in the experiments) or the computed volume of $4 \times 6 \times 0.2 \mathrm{~mm}$ (in the simulations) over a time window of $200 \mathrm{~ms}$.

The calculated size distributions for both models show reasonable agreement with the measurements, as evidenced in Figure 10. In particular, the predictions by O'Rourke-Amsden model for all three cases are in better agreement with the measurements than that done by Bai-Gosman model. The under-estimation by Bai-Gosman model may be due to the reason that though the secondary droplet sizes are sampled by Chi-squared distribution function in the model, the energy conservation is not easy to be guaranteed; especially the dissipative energy loss is considered to be large compared with the incident kinetic energy as shown in Eqs. (18); so the secondary droplet sizes are inclined to be smaller in order to achieve energy conservation. However, the energy conservation has been taken into account directly through droplet sizes sampling in O'Rourke-Amsden model. Because the second argument in Eqs. (12) limits the sizes of secondary droplets at high impact Weber numbers which arises from an energy consistency requirement.

Figure 11 depicts a typical wall film shape in the center slice of the wind tunnel, which has been captured from PLIF image and made from binarization processing. The film thickness is measured between the green line and the red line which stand for the wall surface. The time-averaged thickness was obtained also over a time window of $200 \mathrm{~ms}$.

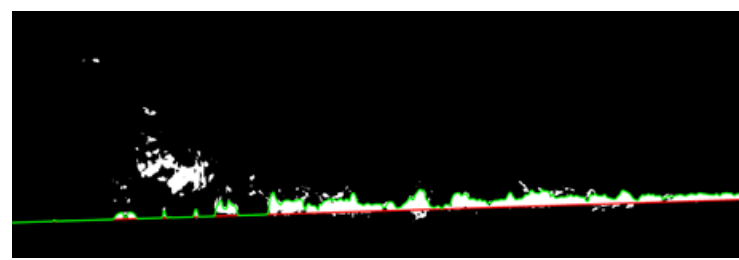

\section{Figure 11 Binary image for measuring wall film thickness}

Figure 12 shows the comparison of measured and predicted time-mean wall film thickness by both models. The results of these two models are fairly close to each other, for the reason that the same wall film model, where the movement of the film is described by a particle-based approach, is used in the presented work. However, we see larger deviations at locations $\mathrm{a}, \mathrm{b}$ and e between modeling and experimental data, while the predicted values at locations $\mathrm{c}$ and $\mathrm{d}$ are acceptable against the measured values. It can be further observed that the film thickness become thinner after location e $(36 \mathrm{~mm}$ downstream from the injector).

The discrepancies exhibited in Figure 12 may be linked to the assumption made in the wall-film model that the velocity profile within the film varies linearly within the film, which is not valid under the high speed cross flow as in our experiments. Besides, the particle-based approach of the model may also be suffered from the uncertainties due to the unsteady particle tracking method for the spray.

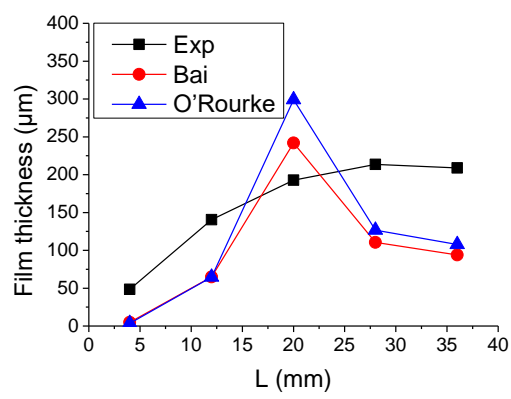

(a) Case 2

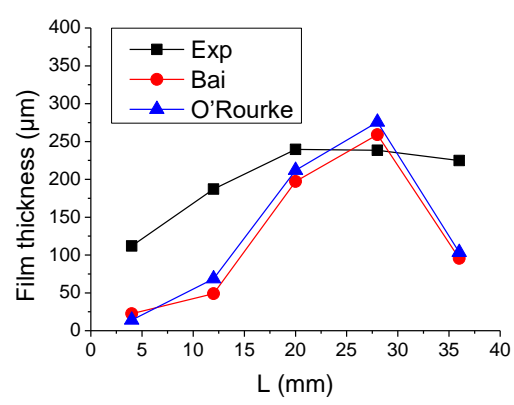

(b) Case 5 


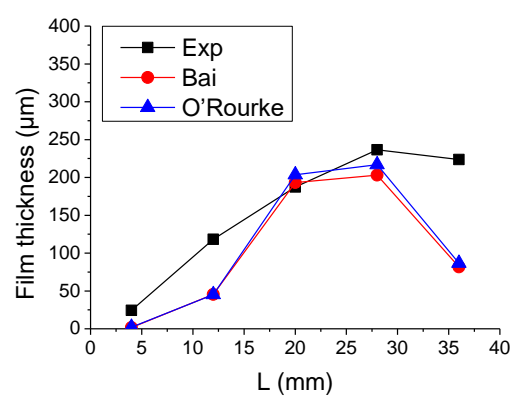

(c) Case 6

\section{Figure 12 Measured and predicted mean wall film} thickness by both models

\section{CONCLUSIONS}

In the presented work, two spray-wall interaction models are implemented into commercial CFD software, ANSYS FLUENT to modeling a series of spray impingement in cross flow which has been conducted experimentally in a wind tunnel. These two models are compared by predicting the droplet sizes and film thickness downstream of the tunnel against the experimental data under different air flow velocities and jet fuel flow rates. For the droplet sizes, the calculated results show in general reasonable agreement with the measurement data. In particular, the predictions by O'Rourke-Amsden model for all three cases are in better agreement with the measurements than that done by BaiGosman model. However, the discrepancies are observed in predicting the film thickness for both models. Thus O'RourkeAmsden model can be used as a sub-model for predicting prefilming atomization, and further work need be done by refining the film movement model and by adding film separation and breakup model in our future research.

\section{NOMENCLATURE}

$\begin{array}{ll}C_{f} & \text { Friction coefficient } \\ d & \text { Droplet diameter } \\ E & \text { Droplet energy } \\ e & \text { Restitution coefficient of rebound velocity } \\ h_{0} & \text { Wall film thickness } \\ L a & \text { Droplet Laplace number } \\ m & \text { Droplet mass } \\ N & \text { Total droplet number } \\ n & \text { Droplet number per parcel } \\ p & \text { Number of secondary droplet parcel per } \\ r & \text { splash } \\ r & \text { Droplet radius } \\ r_{m} & \text { Mass ratio } \\ R e & \text { Droplet Reynolds number } \\ T_{b} & \text { Boiling temperature } \\ T_{w} & \text { Wall temperature } \\ V & \text { Velocity } \\ W e & \text { Droplet Weber number } \\ \alpha & \text { Random number for calculating mass ratio } \\ \delta_{b l} & \text { Boundary layer thickness } \\ \theta & \text { Impingement/splash angle }\end{array}$

$\begin{array}{ll}\rho & \text { Liquid density } \\ \sigma & \text { Surface tension } \\ \psi & \text { Azimuthal angle } \\ \text { Subscript } & \\ \text { crit } & \text { Critical value } \\ D & \text { Dissipative } \\ I & \text { Incident } \\ i & \text { Parcel number } \\ K & \text { Kinetic } \\ N & \text { Normal } \\ S & \text { Secondary } \\ T & \text { Tangential } \\ \sigma & \text { Surface energy }\end{array}$

\section{ACKNOWLEDGMENTS}

This research work is supported by the Innovation Funding project of Aero Engine Corporation of China named as "Pre-filming atomization mechanism and prediction method of swirl cup" with the project No. ZZCX-2017-011. This financial support is gratefully acknowledged.

\section{REFERENCES}

Bai C. and Gosman A. (1995). Development of methodology for spray impingement simulation. In SAE Technical Paper 950283.

Bai C., Rusche H., and Gosman A. (2002). Modelling of gasoline spray impingement. Atomization Sprays 12 (1-3), 128.

Chaussonnet G. (2014). Modeling of liquid film and breakup phenomena in Large-Eddy Simulations of aeroengines fueled by airblast atomizers. $\mathrm{PhD}$ thesis, University of Toulouse.

Ghadiri, H. (1978). Raindrop impact, soil splash and cratering. Ph.D Thesis, University of Reading.

Hsiao G. and Mongia H. C. (2003). Swirl cup modeling part II: inlet boundary conditions. 41th Aerospace Sciences Meeting \& Exhibit, AIAA 2003-1350, 6-9 January.

Matsumoto S. and Saito S. (1970). On the mechanism of suspension of particles in horizontal conveying: Monte Carlo simulation based on the irregular bouncing model. Journal of Chemical Engineering of Japan 3, 83-92.

Mongia H. C., Al-Roub M., Danis A., Lewis D. E., Jeng S. M., Johnson A., MeDonell V. G., Samuelsen G. S. and Vise S. (2001). Swirl cup modeling part I, 37th AIAA/ ASME/SAE/ASEE Joint Propulsion Conference \& Exhibit, AIAA 2001-3576, 8-11 July.

Moreira A., Moita A. and Panão M. (2010). Advances and challenges in explaining fuel spray impingement: How much of single droplet impact research is useful? Progress in Energy and Combustion Science 36 (5), 554-580.

Mundo C., Sommerfeld M. and Tropea C. (1995). Droplet-Wall Collisions: Experimental studies of the deformation and breakup process. International Journal of Multiphase Flow 21(2), 151-173.

Mundo C., Sommerfeld M. and Tropea C. (1998). On the modeling of liquid sprays impinging on surfaces. Atomization Sprays 8 (6), 625-652. 
Mundo C., Tropea C. and Sommerfeld M. (1997). Numerical and experimental investigation of spray characteristics in the vicinity of a rigid wall, Experimental Thermal and Fluid Science 15 (3), 228-237.

Mutchler C. K. (1970). The size, travel and composition of droplets formed by waterdrop splash on thin water layers, $\mathrm{PhD}$ thesis, University of Minnesota.

Naber J. D. and Reitz R. D. (1988). Modeling engine spray/wall impingement. Technical Report 880107, Society of Automotive Engineers. General Motors Research Laboratories, Warren, MI.

Naber J. D. and Reitz R. D. (1989). Modeling engine spray/wall impingement. SAE Trans. 97, 118-140.

O'Rourke P. J. and Amsden A. A. (1996). A particle numerical model for wall film dynamics in port-fuel injected engines. SAE Technical Paper 961961.

O'Rourke P. J. and Amsden A. A. (2000). A spray/wall interaction submodel for the KIVA-3 wall film model, SAE Technical Paper 2000-01-0271.

Stow C. and Hadfield M. (1981). An experimental investigation of fluid flow resulting from the impact of a water drop with an unyielding dry surface. Proceedings of the Royal Society of London. Series A: Mathematical and Physical Sciences 373(1755), 419-441.

Williams, F. A. (1985). Combustion Theory, Benjamin/ Cummings Publishing Company, Menlo Park, CA. 